\title{
Impact of Peak Material Volume of Polycrystalline CVD Diamond Coatings on Dry Friction Against Aluminum
}

\author{
M. PRIESKE ${ }^{1,2}$ \\ 1.-BIAS - Bremer Institut für Angewandte Strahltechnik GmbH, Klagenfurter Str. 5, \\ 28359 Bremen, Germany. 2.—e-mail: bohlen@bias.de
}

For economic and environmental reasons, dry forming is of increasing interest due to the shortening of process chains, cost savings and reduction of environmental pollution. The aim of these investigations is to examine to what extent chemical vapor deposited (CVD) diamond coatings are suitable for dry forming of aluminum and to identify the surface topology requirements for a low friction coefficient and low wear. Nine different surface topologies of CVD diamond coatings were tested in an oscillating ball-on-plate tribometer test against aluminum balls with a Hertzian contact stress of $759 \mathrm{MPa}$ and 99,900 cycles. It could be concluded that the peak material volume (Vmp) of the diamond coating is the most important factor for achieving a low abrasion of aluminum as well as a low friction coefficient against aluminum. The Vmp should be smaller than $0.04 \mathrm{ml} / \mathrm{m}^{2}$. Microcrystalline CVD diamond with a post-treated surface has great potential for dry forming of aluminum.

\section{INTRODUCTION}

Dry forming is of increasing interest for both economic and environmental reasons. Different approaches already lead to a possibility of dry forming. However, tool wear leads to a short tool life, so that dry forming is not yet competitive with oiled forming processes. Due to their high hardness, wear resistance and chemical inertness against aluminum, chemical vapor deposited (CVD) diamond coatings have excellent preconditions as tool coatings for dry forming. Tribological investigations show low coefficients of friction of CVD diamond coatings against different materials. Bhushan et al. ${ }^{1}$ showed a decreasing coefficient of friction with decreasing roughness in the oscillating ball-on-plate test of aluminum balls against differently smoothly polished CVD diamond coatings. This thesis that both the wear rate and the coefficient of friction decrease with a decreasing surface roughness is supported by several publications. Erdemir et al. ${ }^{2}$ concluded, on the basis of tribometer tests of differently rough polycrystalline diamond layers, that the higher the surface roughness, the higher the friction and the wear rate. The high coefficient of friction of rough polycrystalline CVD diamond coatings is attributed to abrasive ploughing and cutting effects of the sharp surface tips. A higher coefficient of friction in the running-in behavior with a subsequent reduction in the coefficient of friction is explained by the breaking-out and flattening of peaks. Erdemir ${ }^{3}$ concluded, based on further publications, that the general rule is that the smoother the diamond surface, the lower the coefficient of friction and the wear rate. Bögli et al. ${ }^{4}$ compared differently treated CVD diamond coatings (mechanically polished, laser polished, plasma polished) with nanocrystalline CVD diamond coatings in a tribometer test. Although the roughness of the surfaces was comparable, the mechanically polished diamond layers [arithmetical mean height (Ra) $35 \mathrm{~nm}$ ] lead to by far the lowest coefficient of friction of 0.03 against monocrystalline ruby. The nanocrystalline CVD diamond coatings with a roughness of $\mathrm{Ra} 30 \mathrm{~nm}$ lead to the highest coefficient of friction of 0.57 . This clearly shows that the roughness $[\mathrm{Ra}$ or the root mean square deviation $(\mathrm{Rq})]$ is not a suitable parameter for establishing a clear correlation with the coefficient of friction and the wear rate.

The aim of this study was to find out whether a surface post-treatment of CVD diamond coatings is necessary for low wear and low friction in dry tribological contact with aluminum or whether a targeted adjustment of the process parameters and the associated crystal size and surface roughness is 
sufficient. The following working hypothesis will be clarified: for mechanically polished and not postprocessed polycrystalline diamond surfaces, a decrease in the wear rate of the aluminum counter body and the coefficient of friction can be predicted with the help of a topology size which takes into account the surface properties responsible for the mechanical clamping and the abrasive wear as a result of a decrease in the topology size.

\section{METHODS}

As substrate material, K10 hard metal discs of the type CTF12D with a diameter of $20.3 \mathrm{~mm}$ and a thickness of $3.0 \mathrm{~mm} \pm 0.15 \mathrm{~mm}$ were used, consisting of $94 \%$ tungsten carbide and $6 \%$ cobalt. The discs where etched by Murakami reagent $\left[\mathrm{K}_{3} \mathrm{Fe}(\mathrm{CN})_{6}: \mathrm{KOH}: \mathrm{H}_{2} \mathrm{O}=1: 1: 10\right]$ for $30 \mathrm{~min}$ and subsequently with Caro's reagent $\left(3 \mathrm{ml} 96\right.$ wt. $\% \mathrm{H}_{2} \mathrm{SO}_{4}$, $88 \mathrm{ml} 40 \% \mathrm{w} / \mathrm{v} \quad \mathrm{H}_{2} \mathrm{O}_{2}$ ) for $60 \mathrm{~s}^{5}$ The diamond nucleation was carried out with a dispersion of $200 \mathrm{ml}$ isopropanol and $210 \mathrm{mg}$ diamond powder with the average crystal size in the range of $0.25 \mu \mathrm{m}$ to $0.50 \mu \mathrm{m}$, from the company Microdiamant AG. The substrates were put into the dispersion within an ultrasonic bath for 10 min and subsequently into isopropanol for $3 \mathrm{~min}$. A laser-based plasma CVD process was used at atmospheric pressure without a vacuum chamber to deposit polycrystalline CVD diamond coatings, which is described in detail in Ref. 6. For the deposition of CVD diamond coatings with different surface topographies, methane and hydrogen with a total flow of 2 standard liters per minute (slm) was added to the argon plasma flame (26 slm). Nine different diamond coatings were deposited by varying the methane/hydrogen ratio, the deposition duration and temperature, which are listed in Table I. The different surfaces of the diamond coatings are shown in Fig. 1. For the designation of the different CVD diamond coatings the mean crystal size is used. The CVDD 10.25p coating was mechanically polished by the company 'Diamond Product Solutions', which is marked by the letter 'p'. In case of the coatings CVDD 8.89r and CVDD 1.91r two samples were rubbed against each other which led to a mechanical polishing of the diamond surface, which is marked by the letter ' $r$ '. The surface roughness parameters of the different CVD diamond coatings are shown in Table II.

Dry tribological oscillating ball-on-plate tests were performed using a CETR Universal Mechanical Tester UMT-3MT. All tests were carried out according to DIN EN 1071-12 standards. A surrounding climate chamber kept the environmental conditions constant at a temperature of $24^{\circ} \mathrm{C} \pm 1^{\circ} \mathrm{C}$ and $40 \% \pm 1 \%$ relative humidity. To examine the long-term behavior of different diamond coatings, a total sliding distance of $1 \mathrm{~km}$ was chosen, corresponding to 99,900 cycles along a linear sliding length of $10 \mathrm{~mm}$. The velocity was set to $50 \mathrm{~mm} / \mathrm{s}$ and the contact force was set to $10 \mathrm{~N} \pm 0.2 \mathrm{~N}$, leading to Hertzian contact pressure of $759 \mathrm{MPa}$, which is in the range of typical sheet metal forming conditions. ${ }^{7}$ The aluminum alloy EN-AW-5083 (AlMg4.5Mn0.7), which is commonly used in the automotive industry, ${ }^{8}$ was used as counter body material. The chemical composition of the aluminum alloy according to the test certificate is shown in Table III.

On one end of an aluminum rod, a hemisphere with a radius of $5 \mathrm{~mm}$ was turned on a lathe, which was used as the counter body. Before testing, the surfaces of the coated plates and round-ended aluminum pins were cleaned with ethanol in an ultrasonic bath for a duration of $5 \mathrm{~min}$. After testing, the wear rates for the round-ended pins were calculated, which is described in detail in Ref. 9. Micro-Raman spectroscopy (Renishaw system 1000) enabled the detection of diamond and graphite by using the excitation wavelength of $514 \mathrm{~nm}$. The existence of diamond was proved by the measurement of the first order Raman line of the diamond at $1332 \mathrm{~cm}^{-1} \cdot{ }^{10}$ The G-band at $1560 \mathrm{~cm}^{-111}$ in the Raman spectra, was utilized to detect graphite in the deposited coatings. Equation $1^{12}$ was used to calculate the diamond quality factors $Q$ of the deposited coatings using the peak intensity of the diamond Raman line $I_{\mathrm{D}}$ and the intensity of the G-band peak $I_{\mathrm{G}}$. The diamond quality factor allows for a semi-quantitative

Table I. Process parameters and sample designation of the different deposited CVD diamond coatings

\begin{tabular}{|c|c|c|c|c|c|c|}
\hline Designation & $\begin{array}{c}\text { Temperature } \\
\left({ }^{\circ} \mathbf{C}\right)\end{array}$ & $\begin{array}{l}\text { Time } \\
\text { (min) }\end{array}$ & $\mathrm{CH}_{4} / \mathrm{H}_{2}(\%)$ & $\begin{array}{l}\text { Thickness } \\
(\mu \mathrm{m})\end{array}$ & $\begin{array}{l}\text { Mean crystal } \\
\text { size }(\mu \mathrm{m})\end{array}$ & Remark \\
\hline CVDD $10.25 p$ & 1050 & 40 & 2 & 14.0 & $10.25 \pm 3.20$ & $\begin{array}{l}\text { Polished, titanium zirconium } \\
\text { molybdenum substrate }\end{array}$ \\
\hline CVDD 9.70 & 1050 & 40 & 2 & 14.6 & $9.70 \pm 3.04$ & \\
\hline CVDD 8.89r & 1050 & 40 & 2 & 14.6 & $8.89 \pm 3.50$ & Rubbed \\
\hline CVDD 4.71 & 1050 & 20 & 2 & 6.0 & $4.71 \pm 0.98$ & \\
\hline CVDD $1.91 \mathrm{r}$ & 900 & 20 & 1 & 3.0 & $1.91 \pm 0.28$ & Rubbed \\
\hline CVDD 1.86 & 900 & 20 & 3 & 3.4 & $1.86 \pm 0.58$ & \\
\hline CVDD 1.66 & 900 & 12 & 3 & 2.0 & $1.66 \pm 0.54$ & \\
\hline CVDD 0.94 & 900 & 12 & 1 & 1.8 & $0.94 \pm 0.26$ & \\
\hline CVDD 0.48 & 750 & 15 & 5 & 1.4 & $0.48 \pm 0.11$ & Nanocrystalline \\
\hline
\end{tabular}



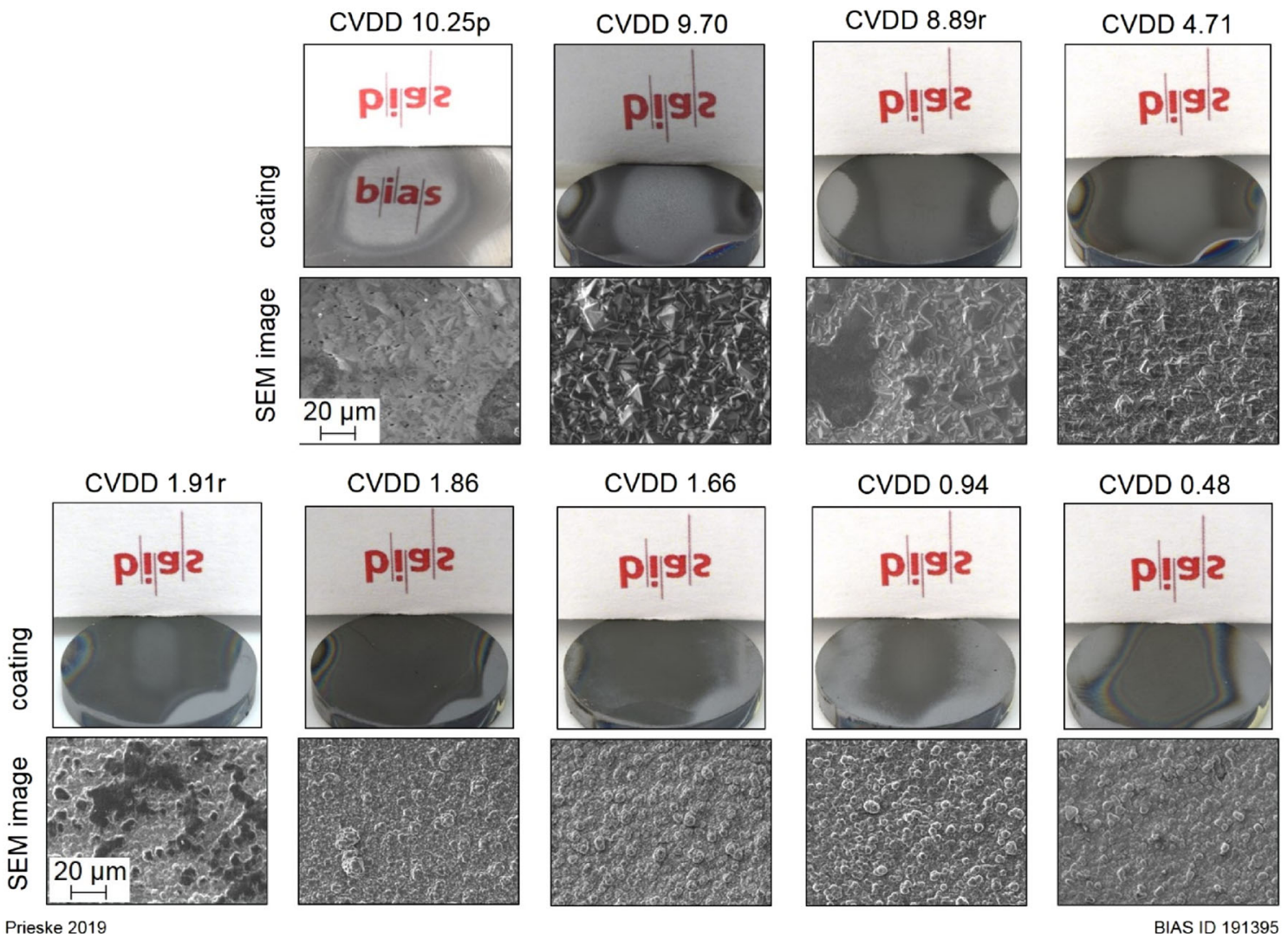

Fig. 1. Different CVD diamond layers used as a plate: photos with reflection of the bias institute logo on top and SE images underneath

Table II. Surface roughness parameters of the deposited CVD diamond coatings according to DIN EN ISO 25178

\begin{tabular}{|c|c|c|c|c|c|c|}
\hline Designation & $\mathbf{S a}(\mu \mathbf{m})$ & Spk $(\mu \mathrm{m})$ & $\operatorname{Sxp}(\mu \mathrm{m})$ & Svk $(\mu \mathrm{m})$ & $\operatorname{Vmp}\left(\mathrm{ml} \mathrm{m}^{-2}\right)$ & $\operatorname{Vmc}\left(\mathrm{ml} \mathrm{m}^{-2}\right)$ \\
\hline CVDD $10.25 p$ & 0.02 & 0.03 & 0.04 & 0.08 & 0.001 & 0.02 \\
\hline CVDD 9.70 & 2.09 & 3.29 & 4.64 & 4.33 & 0.161 & 2.23 \\
\hline CVDD $8.89 \mathrm{r}$ & 1.47 & 1.46 & 2.30 & 3.05 & 0.041 & 1.62 \\
\hline CVDD 4.71 & 1.24 & 1.82 & 2.82 & 2.64 & 0.091 & 1.32 \\
\hline CVDD $1.91 \mathrm{r}$ & 0.84 & 1.02 & 2.03 & 1.37 & 0.051 & 0.93 \\
\hline CVDD 1.86 & 1.27 & 2.11 & 3.13 & 2.63 & 0.106 & 1.33 \\
\hline CVDD 1.66 & 0.94 & 1.41 & 2.39 & 1.69 & 0.070 & 1.01 \\
\hline CVDD 0.94 & 0.85 & 1.33 & 2.19 & 1.38 & 0.066 & 0.92 \\
\hline CVDD 0.48 & 0.68 & 0.95 & 1.66 & 1.14 & 0.047 & 0.74 \\
\hline
\end{tabular}

Table III. Chemical composition of the aluminum alloy EN AW 5083 which was used as the counter body material

\begin{tabular}{llllllllll}
\hline Element & $\mathbf{S i}$ & $\frac{\mathbf{F e}}{0.18}$ & $\frac{\mathbf{C u}}{0.31}$ & 0.04 & $\frac{\mathbf{M n}}{0.67}$ & $\frac{\mathbf{M g}}{4.84}$ & $\frac{\mathbf{C r}}{0.10}$ & $\frac{\mathbf{Z n}}{0.03}$ & $\frac{\mathbf{T i}}{0.02}$ \\
\hline Weight percentage wt.\% &
\end{tabular}

estimate of the quality of a diamond coating, i.e., the concentration of the $s p^{3}$ bonds compared to $s p^{2}$ bonds.

$$
Q=I_{\mathrm{D}} \cdot\left(I_{\mathrm{G}}+I_{\mathrm{D}}\right)^{-1}
$$

Scanning electron microscopy (SEM) images were recorded using an EVO MA-10 (Carl Zeiss). 3D laser microscopy (Keyence VK 9710) was used to measure the depth profiles of the wear tracks and the surface roughness of the different coatings according to DIN EN ISO 25178. 


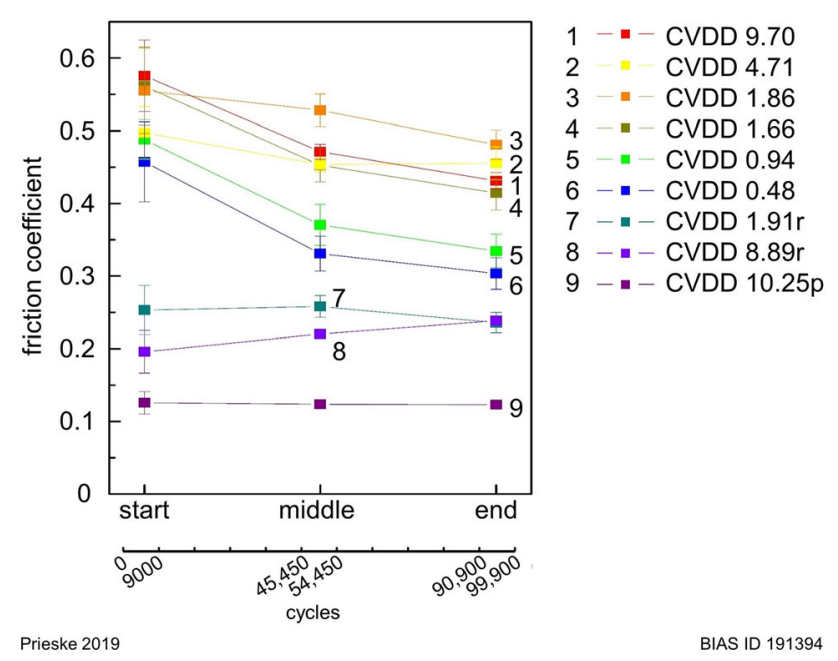

Fig. 2. The friction coefficient values for 9000 cycles are averaged and plotted for the first 9000 cycles, 9000 cycles in the middle of the test, and the last 9000 cycles of tribological examination

\section{RESULTS}

The mean values of the friction coefficients over the first, middle and last half hour (9000 cycles) of the tribometer test are shown in Fig. 2. Of all coatings, the CVDD 10.25p coating shows the most constant behavior over the entire test period. The coefficients of friction of the CVD diamond coatings clearly show the influence of the coating surface in dry tribological contact with aluminum. At the beginning of the tribometer test, the coefficient of friction is between 0.13 and 0.58 depending on the coating surface. In the last half hour of the test, the coefficients of friction of the CVD diamond coatings are between 0.12 and 0.48 .

The wear rates of the aluminum balls after the tribometer tests are shown in Fig. 3. It can be seen that the three diamond coatings with the largest crystal size, which were not post-treated, lead to the largest wear rates of the aluminum balls. The polished diamond coating CVDD 10.25p leads to the smallest wear rate of the aluminum ball of $4.5 \times 10^{-9} \mathrm{~mm}^{3} / \mathrm{Nm}$.

The two coatings with the smallest mean crystal size CVDD 0.48 and CVDD 0.94 show coating delamination at the turning points of the oscillating ball-on-plate test. In Fig. 4 the delamination is shown for CVDD 0.94. This can only be detected at the turning points; in the remaining wear track neither layer detachments nor crack formations of the diamond coating are detected.

The calculated diamond quality factors before and after the tribometer test are shown in Fig. 5. The fact that in each case the diamond quality factor could be determined before and after the tribometer test shows that in no case was the diamond coating worn out in the center of the wear track. The tribometer test does not lead to a change of the

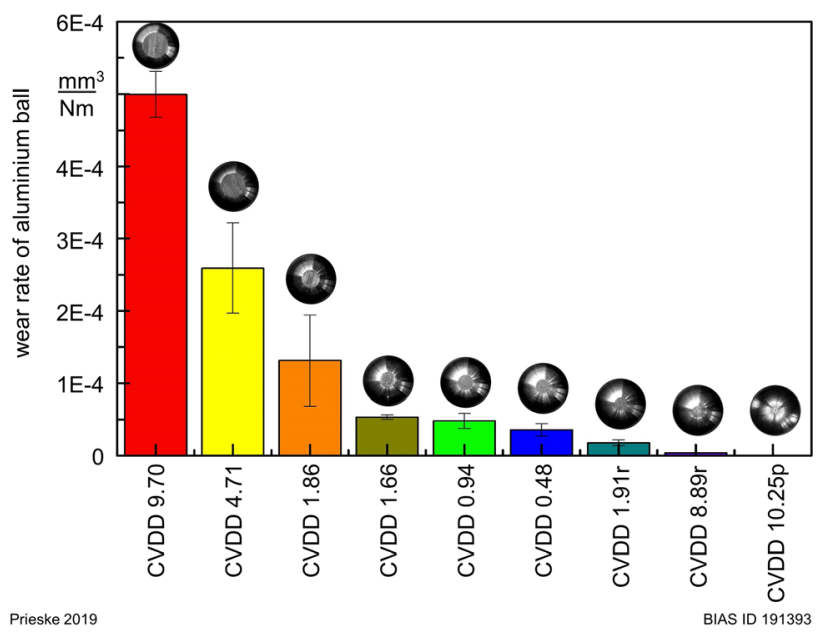

Fig. 3. Wear rate of the aluminum ball after the tribometer test against the different diamond layers as well as an image of the ball after the test

diamond quality factor. Furthermore, it can be seen that the diamond quality factor increases with increasing mean crystal size. The coefficient of friction does not correlate with the diamond quality factor.

Figure 6 shows the coefficients of friction determined in the tribometer test as a function of various parameters for describing the surface roughness [core material volume (Vmc), reduced valley depth (Svk), peak extreme height (Sxp), and reduced peak height (Spk)]. It is noticeable that in all four diagrams, two of the three mechanically polished samples differ from the other samples. These are the two samples which were mechanically polished by rubbing two diamond layers against each other (CVDD 8.89r and CVDD 1.91r). The third mechanically polished coating is the mirror polished coating CVDD 10.25 p, which results in the lowest friction coefficient against the aluminum counter body.

The mean coefficient of friction as function of the arithmetical mean height $\mathrm{Sa}$ as well as of the peak material volume Vmp is shown in Fig. 7. A comparable relationship can be recognized. However, it is noticeable that two mechanically polished coatings are not in line with the relationship in the diagram of the arithmetical mean height. The friction coefficient decreases with a decreasing Vmp and saturates over a Vmp value of $0.1 \mathrm{ml} / \mathrm{m}^{2}$.

The same behavior, that two mechanically polished diamond coatings do not show the relationship of the unpolished coatings, can be seen regarding the wear rate of the aluminum ball as a function of the Sa in Fig. 8a. In contrast, the wear rate of the aluminum ball as a function of the Vmp values (see Fig. 8b) shows a clear correlation for both unpolished and mechanically polished diamond coatings. A decreasing Vmp value leads to a decreasing wear rate down to $0.04 \mathrm{ml} / \mathrm{m}^{2}$. 

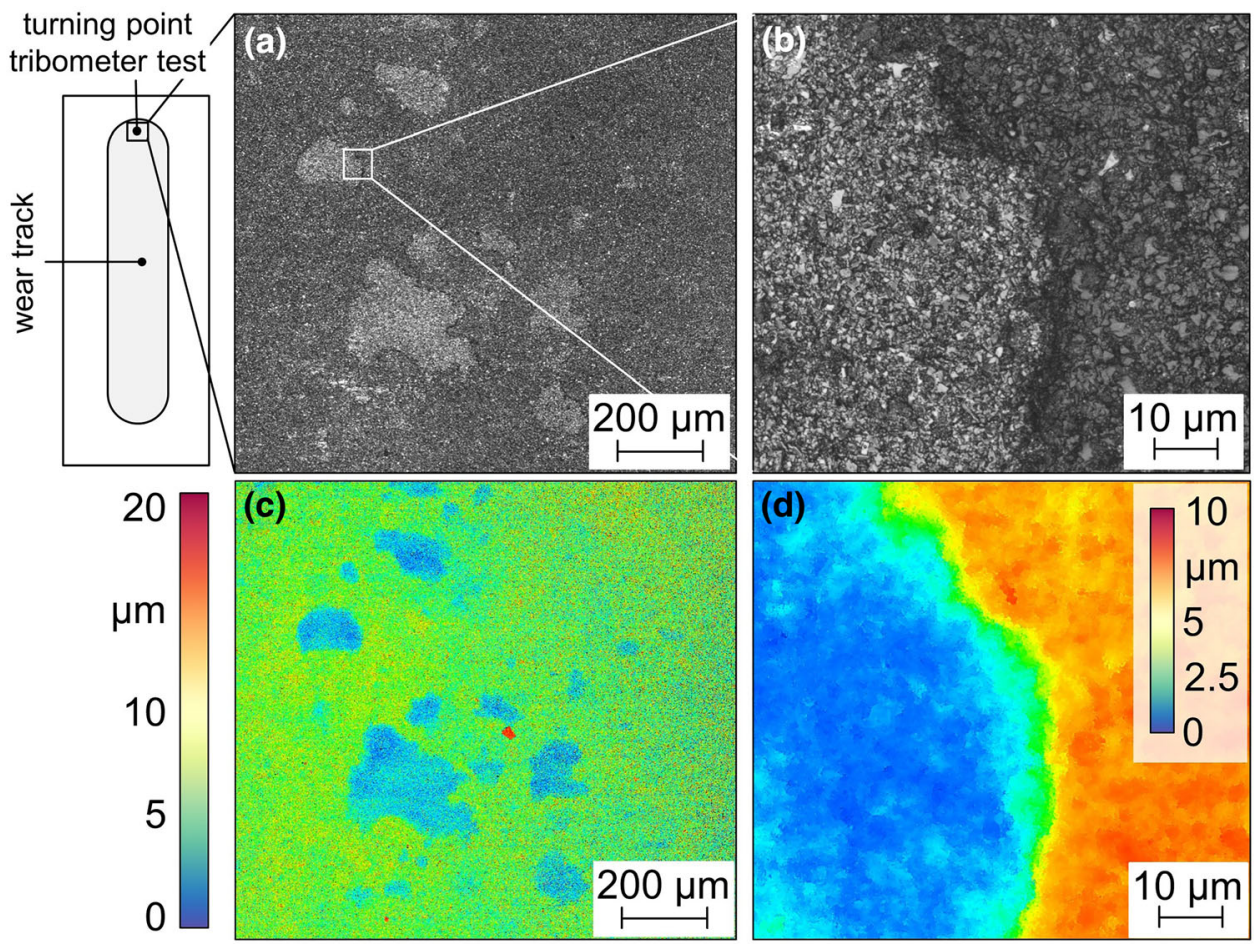

Prieske 2019

BIAS ID 191451

Fig. 4. Coating delamination at the turning point for the CVDD 0.94 coating. (a, b) Confocal microscope images: $b$ is an enlargement of the box marked in (a). (c, d) Height images

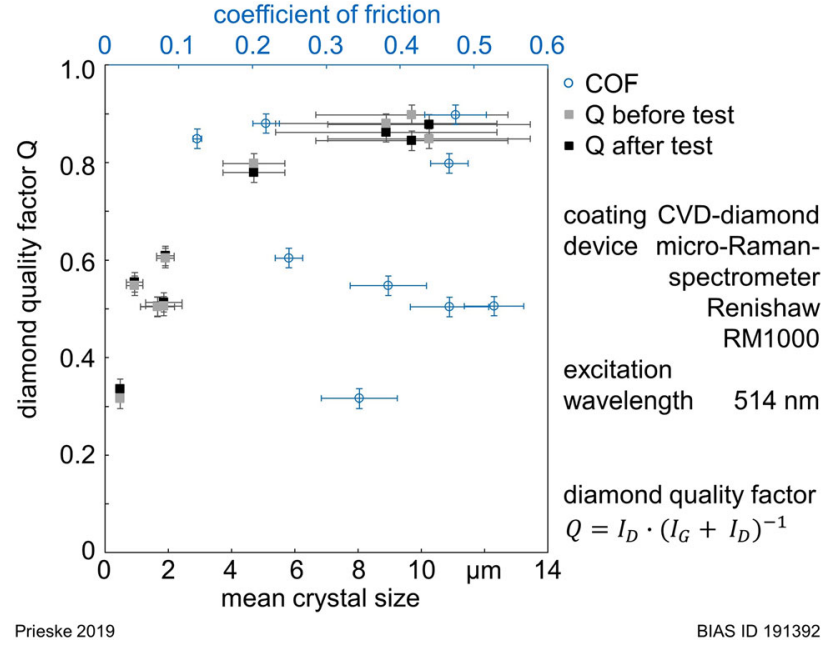

Fig. 5. Diamond quality factor of the CVD diamond coatings before and after the tribometer test plotted against the mean crystal size as well as against the friction coefficient

\section{DISCUSSION}

As described in the introduction, some publications $^{1,3}$ suggest that the lower the surface roughness of the CVD diamond layer, the lower the coefficient of friction and wear rate. Other publications ${ }^{4,13}$ doubt the direct relationship because the relationship is not present, especially in the
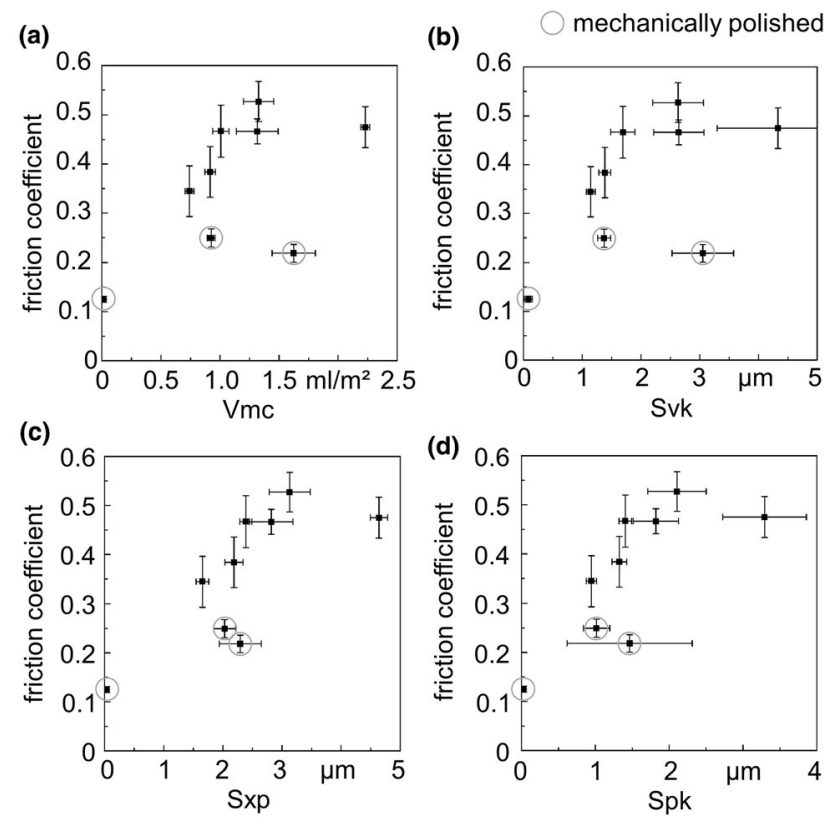

Prieske 2019

BIAS ID 191391

Fig. 6. Mean coefficient of friction as a function of different parameters of surface roughness. (a) Vmc, (b) Svk, (c) Sxp and (d) Spk

comparison between polished and untreated CVD diamond coatings. Hayward et al. ${ }^{13}$ questions the 
(a)

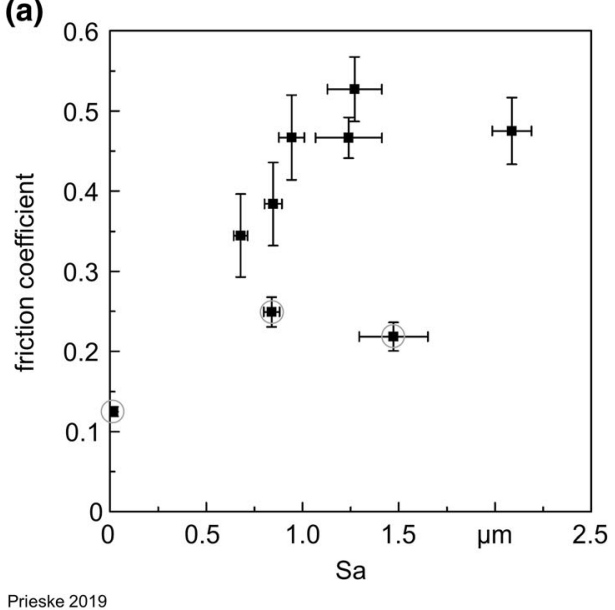

(b)

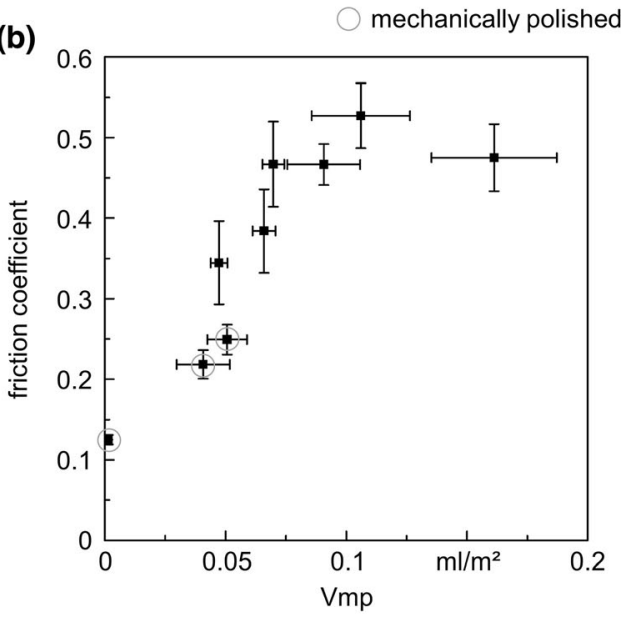

Fig. 7. Mean coefficient of friction as a function of (a) the arithmetical mean height as well as of (b) the peak material volume

(a)

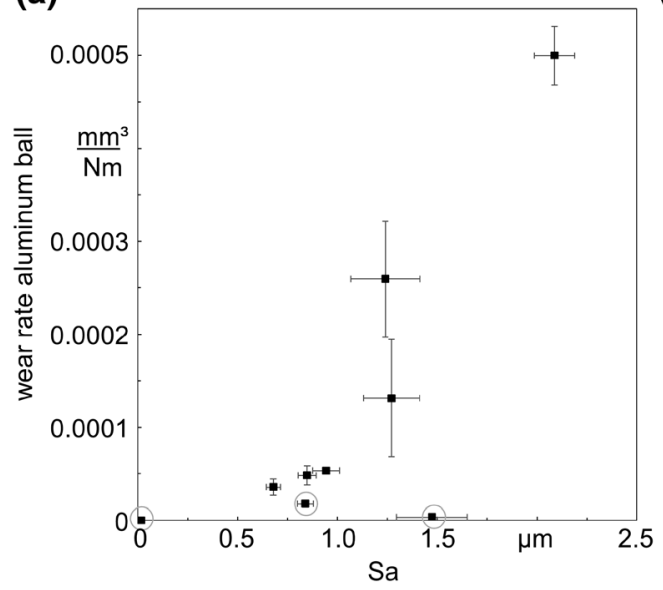

(b)

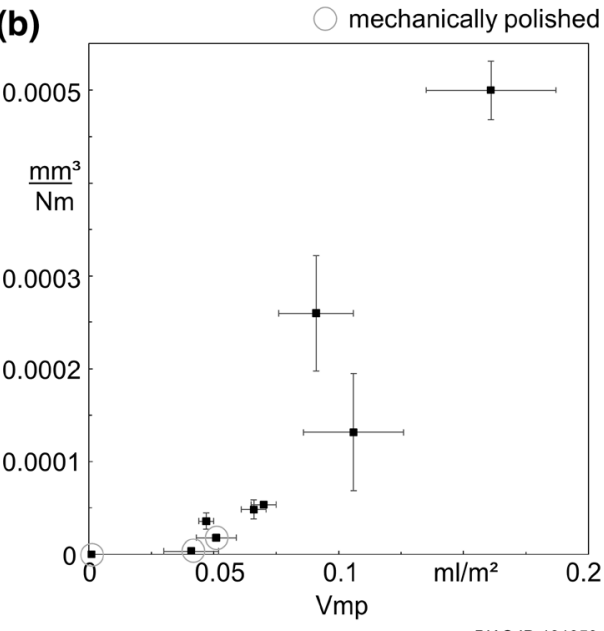

Fig. 8. Wear rate of the aluminum ball as a function of (a) the arithmetical mean height as well as of (b) the peak material volume

measurement method of line roughness. Different measuring tips are used in different publications, which leads to different results for the same surfaces. In this work, instead of line roughness, surface roughness was measured with a laser scanning confocal microscope. This enables the evaluation of a large number of other surface roughness parameters.

The surface of the untreated CVD diamond layers differs mainly in the different crystal sizes. This leads to a scaling of most surface roughness parameters. The mechanically polished CVDD 10.25p coating achieves low values for all parameters due to the mirror-polished surface, so that in the diagrams it fits into the course of a decreasing coefficient of friction due to decreasing surface roughness parameters. The rubbed CVD diamond coatings lead to inhomogeneous roughness parameters. The deep valleys of the microcrystalline CVD diamond layer are still present, but the protruding peaks have been greatly reduced, resulting in plateaus. The difference becomes particularly clear in Fig. 7 when comparing the CVDD 9.70 and the CVDD 8.89r coatings. Rubbing two CVD diamond coatings with a mean diamond crystal size of $8.89 \mu \mathrm{m}$ against each other reduces the mean arithmetic height, Sa, from $2.09 \mu \mathrm{m}$ to $1.47 \mu \mathrm{m}$ (by $30 \%$ ), which is still the second highest value compared to the other coatings (Fig. 7a the two values with the highest Sa). The peak material volume, however, was reduced from $0.161 \mathrm{ml} / \mathrm{m}^{2}$ to $0.041 \mathrm{ml} / \mathrm{m}^{2}$ (by $75 \%$ ) and thus from the second highest to the second lowest value (see Fig. 7b). In the diagram of the coefficient of friction as a function of the Vmp, the correlation can be seen that an increase in the Vmp leads to a near-to-linear increase in the coefficient of friction. From a Vmp value of about $0.1 \mathrm{ml} / \mathrm{m}^{2}$, the coefficient of friction becomes saturated at about 0.5. Furthermore, the diagram shows that it is not to be expected that the coefficient of friction between the aluminum alloy EN AW 5083 and diamond will fall below 0.1. 
From the diagram in Fig. 8 it can be concluded that the volume of the top $10 \%$ of the surface to which the peak material volume (Vmp) refers is the relevant factor for the abrasion of aluminum. The hypothesis, "For mechanically polished and nonpolished polycrystalline diamond surfaces, a decrease in the wear rate of the aluminum counter body and the coefficient of friction due to a decrease in the topology size can be predicted with the help of a topology size which takes into account the surface properties responsible for the mechanical interlocking and the abrasive wear," could be confirmed. The topology size searched for, which for non-polished and mechanically polished CVD diamond coatings is equally capable of estimating the coefficient of friction as well as the wear rate of the aluminum counter body in the dry oscillating ball-on-plate tribometer test, is the peak material volume.

For diamond coatings in dry contact with the aluminum alloy EN AW-5083, it can be concluded from the investigations that in order to minimize the wear rate of the aluminum counter body, a peak material volume of less than $0.04 \mathrm{ml} / \mathrm{m}^{2}$ should be aimed for. The two untreated CVD diamond coatings with the lowest Vmp values (CVDD 0.48 and CVDD 0.94) exhibit layer delamination at the turning points. This means that they do not withstand repeated static friction with the chosen coating properties. For this reason, without further coating process development, the deposition of microcrystalline CVD diamond layers with subsequent post-treatment of the surface is recommended in order to achieve a peak material volume of less than $0.04 \mathrm{ml} / \mathrm{m}^{2}$ for dry piece goods processes of aluminum workpieces.

\section{CONCLUSION}

The peak material volume (Vmp) is equally capable of estimating, for non-polished and mechanically polished CVD diamond coatings, the coefficient of friction as well as the wear rate of the aluminum counter body in the dry oscillating ballon-plate tribometer test. For dry piece goods processes of aluminum workpieces, the deposition of microcrystalline CVD diamond layers with subsequent post-treatment of the surface is recommended in order to achieve a peak material volume of less than $0.04 \mathrm{ml} / \mathrm{m}^{2}$ in order to minimize the wear rate of the aluminum counter body. The lower the Vmp value, the lower the coefficient of friction, so that a minimum Vmp value must be achieved in the case of a desired minimum coefficient of friction.

\section{ACKNOWLEDGEMENTS}

Open Access funding provided by Projekt DEAL. This work was supported by Deutsche Forschungs- gemeinschaft (DFG) under the Project Number 390771352, which the authors gratefully acknowledge.

\section{CONFLICT OF INTEREST}

The author declares that he has no conflict of interest.

\section{OPEN ACCESS}

This article is licensed under a Creative Commons Attribution 4.0 International License, which permits use, sharing, adaptation, distribution and reproduction in any medium or format, as long as you give appropriate credit to the original author(s) and the source, provide a link to the Creative Commons licence, and indicate if changes were made. The images or other third party material in this article are included in the article's Creative Commons licence, unless indicated otherwise in a credit line to the material. If material is not included in the article's Creative Commons licence and your intended use is not permitted by statutory regulation or exceeds the permitted use, you will need to obtain permission directly from the copyright holder. To view a copy of this licence, visit $h$ ttp://creativecommons.org/licenses/by/4.0/.

\section{REFERENCES}

1. B. Bhushan, V.V. Subramaniam, A. Malshe, B.K. Gupta, and J. Ruan, J. Appl. Phys. 74, 4174 (1993).

2. A. Erdemir, G.R. Fenske, C. Bindal, C. Zuiker, A.R. Krauss, and D.M. Gruen, in 6th European Conference on Diamond, Diamondlike and Related Materials, vol. 1 (1995).

3. A. Erdemir, Proc. Inst. Mech. Eng. Part J J. Eng. Tribol. 216, 387 (2002).

4. U. Bögli, A. Blatter, S.M. Pimenov, E.D. Obraztsova, A.A. Smolin, M. Maillat, A. Leijala, J. Burger, H.E. Hintermann, and E.N. Loubnin, Diam. Relat. Mater. 4, 1009 (1995).

5. L. Wang, X. Lei, B. Shen, F. Sun, and Z. Zhang, Diam. Relat. Mater. 33, 54 (2013).

6. M. Prieske, S. Müller, and P. Woizeschke, Coatings 9, 537 (2019).

7. M.P. Pereira, W.Y. Yan, and B.F. Rolfe, Mater. Sci. Forum 561-565, 1975 (2007).

8. Aluminium-Magnesium 5083, http://www.sabater-fundim ol.com/customers/resources/pdf/sabater-fundimol_catalog_p 28-29.pdf, Accessed 25 Oct 2018.

9. M. Prieske, H. Hasselbruch, A. Mehner, and F. Vollertsen, Surf. Coat. Technol. 357, 1048 (2019).

10. A.C. Ferrari and J. Robertson, Philos. Trans. Ser. A Math. Phys. Eng. Sci. 362, 2477 (2004).

11. A.C. Ferrari and J. Robertson, Phys. Rev. B 64, 199 (2001).

12. K.S. Pal, A.K. Mallik, N. Dandapat, N.R. Ray, S. Datta, S. Byakh, and B.K. Guha, Bull. Mater. Sci. 38, 2 (2015).

13. I.P. Hayward, Surf. Coat. Technol. 49, 554 (1991).

Publisher's Note Springer Nature remains neutral with regard to jurisdictional claims in published maps and institutional affiliations. 